\title{
Riscrivere la storia coloniale tramite l'uso dell'oralità: Il caso di Adua (2015)
}

\author{
Anna Finozzi \\ Stockholms Universitet
}

\begin{abstract}
:
The article examines the use of orality in Igiaba Scego's novel Adua (2015). Traditionally, the postcolonial literary text is regarded as a 'translation' between two languages: the African oral one and the European written one. The aim of the article is to move the attention from orality as a sign of Otherness towards orality as transmission and re-telling; this critical shift is necessary as Italian postcolonial literature is often regarded more as a documentary text rather than a literary artefact. By relying on Memory Studies, and specifically on concepts such as Marianne Hirsch's postmemory, Yael Zerubavel's countermemory and Astrid Erll's travelling memory, the analysis shows that $A d u a$ is built on the oral communication of memories through dialogues between different characters and other images connected to the act of listening and retelling. Eventually, the dichotomy orality-africanity is dismissed in favour of orality-transmission.
\end{abstract}

Keywords: Italian postcolonial literature; orality, memory studies; cultural memory

\begin{abstract}
:
L'articolo si propone di analizzare l'uso dell'oralità nel romanzo Adua (2015) di Igiaba Scego. Tradizionalmente, il testo letterario postcoloniale è stato considerato come una 'traduzione' da una lingua orale Africana ad una scritta Europea. Lo scopo dell'articolo è spostare l'attenzione dall'oralità come segno di alterità all'oralità come modalità di trasmissione; questo slittamento critico è necessario per una rivalutazione della letteratura postcoloniale italiana, di cui spesso si considera più la portata documentaristica di quella letteraria. Attraverso i Memory Studies, e in particolare concetti quali la postmemory di Marianne Hirsch, la countermemory di Yael Zerubavel e la travelling memory di Astrid Erll, l'analisi mostra come Adua sia modellata dalla comunicazione orale della memoria attraverso i dialoghi dei personaggi e da altre immagini connesse all'atto di ascoltare e tramandare. Infine, la dicotomia oralità-africanità viene respinta in favore di quella oralità-trasmissione.
\end{abstract}

Parole chiave: Letteratura italiana postcoloniale; oralità; memory studies; memoria culturale 
A partire dagli anni sessanta, l'uso delle lingue pre-coloniali di tradizione orale e la dirompenza dell'Alterità nel testo ibrido di lingua "eurofona" (anglofona e francofona specialmente) contribuirono alla politica di decolonizzazione culturale delle neoindipendenze. ${ }^{1}$ L'intromissione di diversi artifici formali (a livello morfo-sintattico e lessicale) e di suggestioni folkloristiche e proverbiali è stata quindi percepita come la traduzione di un bagaglio culturale orale "africano" nella letteratura europea di arrivo, come motivo ideologico (contro-canone) ed estetico (Bandia, Translation 218). ${ }^{2}$ Ma che funzione possiamo attribuire oggi alla presenza dell'oralità nei testi di seconda generazione nella letteratura italiana postcoloniale? ${ }^{3}$

L'oralità non interferisce solamente sulla grammatica, ma pervade e intesse la struttura narrativa. Non solo, costituisce in molti casi la base del testo scritto; basti pensare alla prima fase della letteratura della migrazione in cui i giornalisti trascrivevano i ricordi degli immigrati e ne rielaboravano le memorie orali, o all'impalcatura portante di un romanzo come Regina di fiori e di perle (2007) di Gabriella Ghermandi, "un lavoro durato mesi, in cui l'autrice ha ascoltato, registrato e trascritto le storie di tante persone" (Lombardi-Diop 308). Focalizzarsi sulle modalità di trasmissione significa utilizzare la lente dei memory studies. ${ }^{4}$ Infatti, la memoria, considerata tradizionalmente una forma orale del raccontare, viene a contatto con la letteratura, sfumando i confini tra storia e fiction e creando quindi quei lieux de mémoire in cui è possibile rintracciare le molteplici direzioni del passato (Nora 24).

Il mio intervento si propone di analizzare il caso del romanzo Adua di Igiaba Scego, pubblicato da Giunti nel 2015, e di dimostrare come l'oralità sia una strategia narrativa fondamentale per la diffusione di una conoscenza coloniale e per la formazione di una coscienza postcoloniale in Italia. Adua convoglia ed esplora i flussi migratori contemporanei, la storia della Somalia coloniale e uno spaccato dell'AFIS ${ }^{5}$, la Roma fascista prima dell'arrivo delle leggi razziali, la figura del corpo nero femminile esotizzato e sessualizzato in Occidente e il ruolo del cinema nel suo processo di stereotipizzazione. L'oralità sottende tutto il volume: da una parte, infatti, il testo è costruito su una corale di conversazioni ascoltate e non ascoltate, sui racconti riportati dai padri ai figli e alle figlie, sull'attività di traduttore di Zoppe, sulla mediazione e rimediazione dell'eredità coloniale, sul ricordo dell'infanzia, sul resoconto di Adua e sull'invettiva disperata della Paternale. Dall'altra, le espressioni orali in somalo, segnalate dal corsivo, graffiano il testo italiano, creando quell'ibridismo tipico del linguaggio letterario del romanzo postcoloniale, inserito a enfatizzare i canali di propagazione della memoria.

\footnotetext{
${ }^{1}$ A questo proposito, si può ricordare ad esempio il dibattito iniziato nel 1962 ad un convegno al Makerere University College in Uganda per celebrare l'emergere della nuova letteratura africana in inglese. Per una panoramica: Gikandi.

2 Tutte le traduzioni dall'inglese sono mie, se non diversamente specificato.

${ }^{3}$ Una precisazione: con "letteratura postcoloniale italiana" mi allaccio alla definizione rimarcata da Caterina Romeo in un recente articolo in cui con "postcoloniale" non si intende solo la letteratura scritta da autori nati o discendenti dalle ex-colonie italiane, ma anche dalle altre colonie europee ("postcoloniale diretto" e "postcoloniale indiretto"). Con "letteratura della migrazione," invece, si può intendere la fase iniziale (anni novanta) della produzione postcoloniale, fase in cui gli scritti dei migranti erano spesso frutto di collaborazioni con giornalisti italiani e di stampo autobiografico. Rimando a: Romeo.

${ }^{4}$ Nell'introduzione al loro The collective memory reader, Jeffrey K. Olick, Vered Vinitzky-Seroussi e Daniel Levy hanno tracciato le linee della storia dei Memory Studies dal memory boom di fine anni settanta.

5 “Amministrazione fiduciaria italiana della Somalia" (1950-1960).
} 


\section{Oralità e testo postcoloniale: la questione della lingua}

Nell'ambito degli studi letterari, sono stati coniati diversi termini per riferirsi al processo di commistione linguistica operata nel testo postcoloniale: oraliture ${ }^{6}$ ibridità, ${ }^{7}$ eteroglossia, ${ }^{8}$ métissage e altri. Nonostante sia stato contestato ampiamente l'utilizzo del termine "Oralità" per riferirsi a un testo scritto, ${ }^{10}$ continuare ad adoperarlo significa ricordare quello sforzo orientalista "di salvataggio" esercitato dagli europei a favore delle culture non civilizzate (perché non erette su testi scritti) e, di conseguenza, a quella mania di collezionare esemplari di pensiero locali, misteriosi ed esotici (Barber 20). ${ }^{11}$

"Romanzare l'arte orale porta alla creazione di un discorso ibrido che richiede una strategia di lettura-come-traduzione, richiamando così l'attenzione sulla natura traduttiva del testo postcoloniale" (Bandia, Translation 53). In questo senso, dalla "svolta culturale" ("cultural turn") degli anni ottanta, i Translation Studies hanno avuto un ruolo cruciale nell'analisi del testo postcoloniale. Paul Bandia nel suo Translation as reparation (2008) ha approfondito le diverse tecniche adoperate nella commistione delle lingue orali africane con l'inglese e il francese in Things fall apart (1958) del nigeriano Chinua Achebe, Une vie de boy (1956) del camerunense Ferdinand Oyono e The voice (1964) del nigeriano Gabriel Okara. Alcune delle strategie che ha discusso sono: l'inserimento di proverbi ("Nous mangions en silence car la bouche qui parle ne mange pas," Une vie de boy), l'uso di calchi sintattici dalla lingua orale ("Who are you people be?," The voice), gli spostamenti semantici ("figlio" invece di "cugino" senza considerare la relazione biologica), il code-switching (accostamento di due lingue in una frase o in un paragrafo), i termini in-between ("this never-happened-before-thing," The voice), le ripetizioni ("Black black night," The voice), il cushioning ("He is Okonkwo kpom-kwem, exact, perfect," Things Fall Apart, termine europeo seguito subito dopo da quello vernacolare per renderlo comprensibile).

Anche nel testo italiano, la lingua viene manomessa con degli inserimenti provenienti dal bilinguismo degli autori migranti e dall'effetto che esso ha sull'italiano

\footnotetext{
${ }^{6}$ Daniele Comberiati (Comberiati, Scrivere) ha utilizzato il termine oraliture, dalla contrazione di "oralité" and "écriture," citando Chamoiseau.

7 "Ibridità" ("Hybridity") negli studi postcoloniali fa capo alle teorie di Homi Bhabha sulla resistenza e la sovversione (Bhabha). Come ha scritto Andrew Smith, dopo Bhabha il termine "ibridità," entrato inizialmente nel gergo comune come peggiorativo ("non puro"), si è poi avvicinato ad un significato diverso, per indicare "la qualità delle narrative e dei discorsi" (225).

8 "Eteroglossia" ("Heteroglossia") è stato usato da Amitav Ghosh per indicare le appropriazioni e le variazioni dell'inglese ad opera degli autori della diaspora indiana. Altri due testi che trattano lo stesso argomento: Sharmani; Bandia, "Postcolonial".

9 "Métissage" è stato utilizzato da Françoise Lionnet per riferirsi alle lingue creolizzate, adoperate nelle zone francofone decolonizzate e nelle zone di confine. Scrive Lionnet: "In quegli spazi, le lotte per il controllo dei mezzi di rappresentazione e auto-identificazione sono mediate da un sistema simbolico unico e immensamente potente: la lingua coloniale, e le variazioni alle quali questa è soggetta per merito degli scrittori che la arricchiscono, la trasformano e la creolizzano.” (Lionnet 101)

${ }^{10}$ Walter Ong ha scritto che definire le tradizioni e tutto ciò (generi, stili) che riguarda l'oralità come "letteratura orale" è come pensare "ai cavalli come ad automobili senza ruote" (Ong 12).

${ }^{11}$ Scrive Karin Barber: "Historians, and more generally the nineteenth century diplomats, travellers and administrators who were the cultural wing of imperial conquest, were deeply interested in native texts. [...] Creating written texts out of oral traditions was an effort of salvage, an act of redemption, a building of the groundwork on which a civilised culture could be erected, as well as the collecting of a range of specimens of local, customary ways of acting and thinking" (Barber 20).
} 
parlato. ${ }^{12}$ Un esempio è il "portuliano" nel brano "Ana de Jesus" dell'italo-brasiliana Christiana de Caldas Brito, in Amanda Olinda Azzurra e le altre (1998). Il racconto mima una parlante straniera che, per rivolgersi alla "signora," utilizza una lingua diversa dall'italiano standard. Nella frase: "Se i piedi sono senza escarpe e i diti sono felici di pestare la terra, perché mia bambina deve usare escarpe?" (31) si nota subito, ad esempio, la "e" che funge da vocale di appoggio, tipico dell'area ispanica e lusofona. Il senso della parlata orale viene enfatizzato anche dall'uso massiccio di onomatopee, come ad esempio "tom tom tom tom, l'orologio batte" (33), e ripetizioni "Cani del mio paese magri, magri" (30), racchiuso infine nella riflessione "metalinguistica" con cui Ana chiude il racconto: "Qui non so parlare il nome mio. Quando dico Ana de Jesus le persone mi corregge e dice un altro nome che non è il mio. Ana diventa Anna, Jesus diventa Gesù” (33).

Scrive Lucia Quaquarelli:

La lingua letteraria diventa strumento di appropriazione che si oppone all'assimilazione facendosi resistenza, rivolta e trasgressione; la lingua del padrone viene rimaneggiata, reinventata, assorbita e rimodellata, divorata e poi risputata nuova, e la lingua madre può di nuovo prendere la parola, dentro la lingua dell'altro, nelle sue viscere (Quaquarelli 75).

Nonostante la riappropriazione della lingua di arrivo e l'innesto di Alterità rimangano i ruoli fondamentali della sperimentazione formale nel contesto postcoloniale e migrante, a mio avviso i motivi che ne sottendono l'utilizzo hanno ragioni diverse, soprattutto nel contesto italiano, in cui la letteratura postcoloniale ha avuto il suo momento più "letterario" con le seconde generazioni (Romeo 12).

La spinta disgregatrice e anti-canonica appartiene, infatti, a una generazione di scrittori che usciva dal colonialismo e cercava di riaffermare la propria ritrovata identità culturale e nazionale, e agli autori della diaspora, emigrati in un'Europa e in un'Italia in cui lo straniero molto spesso è relegato ai margini della vita culturale, sociale e politica. Quando ci si riferisce alle seconde generazioni, si tratta di autori che, come Igiaba Scego, sono nati in Italia o si sono trasferiti in Italia molto giovani; autori cioè che non hanno avuto nella loro formazione, o l'hanno avuta parzialmente, un'educazione scolastica in un paese diverso dall'Italia. Il loro legame con la terra di origine sembra quindi basato sul racconto orale, sulle storie e i resoconti all'interno del circuito famigliare o della comunità diasporica. Come ha ricordato Ubax Cristina Ali Farah riferendosi alle strategie della sua letteratura, "la vicinanza con l'oralità mi serve soprattutto per ricreare il ritmo, la sequenzialità narrativa, per far emergere l'umorismo e il modo somalo di raccontare. In un certo senso unisco queste mie due dimensioni linguistiche scrivendo in italiano storie che ascolto in somalo" (Comberiati, La quarta 48).

La trasmissione, infatti, non coinvolge la volontà di preservare una lingua orale dall'egemonia di una lingua scritta più potente (come nel caso dell'inglese), né quella di esportarla in altri contesti (come nel caso dei romanzi africani pubblicati in Europa), ma concerne l'ascolto e la riscrittura di storie che sono state comunicate oralmente e poi rielaborate su carta e che, quindi, ne mantengono letterariamente l'influenza colloquiale e gli influssi non italiani. Ne è un esempio Regina di fiori e di perle (2007) nella cui postfazione Cristina Lombardi-Diop scrive: "A differenza di quello dello storico, il cui lavoro di selezione e interpretazione delle fonti risponde a un principio di attendibilità,

\footnotetext{
${ }^{12}$ Con "autori migranti" mi riferisco agli autori di prima generazione.
} 
il processo di selezione e d'indagine della Ghermandi risponde alle strategie letterarie e alle esigenze della forma narrativa orale. L'oralità è il principio strutturante del romanzo" (309). In questo modo, si riesce anche ad abbandonare la ricerca dell'“autenticità africana" (tradizionalmente riscontrabile nell'effetto orale "in aggiunta" al testo europeo), come indice di "primitività o pienezza," e a connettere l"“africanità" alle modalità di trasmissione della memoria e all'impianto colloquiale delle conversazioni romanzate all'interno del testo (Julien 162).

\section{Il ruolo della memoria}

Come ha scritto Aleida Assmann, "negli ultimi due decenni, la storia ha ricevuto un potente rivale o partner nella sua pretesa di accedere, ricostruire e rappresentare il passato, cioè la memoria" (262). La memoria era stata infatti ritenuta come una pericolosa distorsione dei fatti veri rinvenuti dalla storiografia per la sua natura fallace e frammentaria, e, nelle parole di Pierre Nora, per essere "aperta alla dialettica del ricordare e dimenticare, [...] vulnerabile alla manipolazione e all'appropriazione" (8). Nonostante questo, oggi "storia e memoria $[\ldots]$ non sono più considerate rivali e sono sempre più accettate come modi complementari di ricostruire [...] il passato" (A. Assmann 263). La memoria è quindi divenuta sostanziale nella produzione di conoscenza storica e, di conseguenza, i testimoni hanno iniziato ad avere un peso decisivo nell'investigazione e nella trasmissione. Uno degli eventi che ha tristemente segnato il passaggio verso il genere della testimonianza, prima confinato alla sfera famigliare o a gruppi ristretti, è stato il processo di Eichmann (1961) in cui centoundici sopravvissuti testimoniarono le atrocità subite (Wieviorka 390-391).

Jan Assmann distingue la "memoria comunicativa" dalla "memoria culturale" (209). La memoria comunicativa, della durata massima di ottant'anni, è la memoria biografica trasmessa quotidianamente o quasi all'interno di un gruppo famigliare. Nel passaggio da una generazione all'altra, Marianne Hirsch ha anche parlato di post-memory, cioè la struttura di trasmissione inter- e trans-generazionale della conoscenza traumatica, "relazione che la generazione seguente a coloro che hanno assistito a un trauma culturale o collettivo mantiene con chi è venuto prima, esperienze che 'ricordano' solo attraverso storie, immagini e comportamenti in mezzo a cui sono cresciuti" (106). La trasmissione della memoria non avviene solamente tramite il linguaggio ma anche attraverso "atti non-verbali e non-cognitivi" all'interno della famiglia (112). A livello narrativo quello tra Adua e Zoppe, come illustrerò tra poco, è un esempio di come il trauma si perpetri nella seconda generazione.

La memoria culturale ha una durata maggiore e delinea l'identità di un gruppo più vasto. È costituita da forme simboliche che annullano la distinzione tra "mito" e "storia" (J. Assmann 210). In questo senso, la memoria culturale è in forma narrativa, una narrativa che fa leva sui sistemi creati dal gruppo sociale da cui proviene:

la memoria non è semplicemente il deposito dei "fatti" ma un continuo lavoro di immaginazione ricostruttiva ("reconstructive imagination"). In altre parole, il passato non può essere immagazzinato ma deve essere sempre "processato" e mediato. Questa mediazione dipende da cornici semantiche e bisogni di determinati individui e società in un determinato presente. (J. Assman 210) 
I testi letterari, quindi, come altre forme di mediazione, hanno un ruolo centrale nella propagazione della memoria. ${ }^{13} \mathrm{La}$ forte componente orale della letteratura postcoloniale italiana risente dei processi di ricordo e riorganizzazione delle memorie che trascendono il contesto famigliare per elevarsi a "memoria culturale." Siamo quindi di fronte a quel tentativo di riscrittura della storia ufficiale del colonialismo italiano che, come ci informa Angelo Del Boca, riguardava inizialmente solo una serie di cinquanta volumi scritti per opera del Ministero degli Affari Esteri, definita da Del Boca stesso come un "colossale, dispendioso, quasi incredibile sforzo di mistificazione" (590).

Come ha scritto Caterina Romeo:

La memoria è un elemento centrale nei testi di Cristina Ali Farah, Gabriella Ghermandi e Igiaba Scego, non soltanto perché serve per riscrivere la storia personale degli individui e collettiva dei popoli, ma anche perché il modo in cui gli eventi vengono pubblicamente ricordati (o dimenticati) mostrano in che modo una nazione vuole costruire la propria memoria storica e quindi la propria identità (Romeo 2011, 404).

Quella che Yael Zerubavel, riprendendo un termine coniato da Michel Foucault, ha definito come countermemory, cioè "la narrativa commemorativa alternativa che si oppone direttamente alla narrativa ufficiale," diventa quindi l'insieme di testimonianze raccolte e rimaneggiate dagli scrittori e dalle scrittrici postcoloniali nel topos del racconto all'interno dei loro testi. In Regina di fiori e di perle la protagonista viene addirittura investita del ruolo di cantora del suo popolo. ${ }^{14}$

Prima di partire per la Germania, Ahmed, il giovane marito di Adua, le regala una telecamera, simbolo di un passato tormentato dietro un'impietosa cinepresa e della possibilità di potersi ricostruire da zero: "ora potrai filmare quello che vuoi, ora potrai narrarti come ti pare e piace" (174). Adua, come si vedrà tra poco, è uno di quei romanzi che rivelano l'intento di portare al vaglio, di ri-visitare e ri-raccontare la storia non-ufficiale, quella dei più deboli, quella rimasta esclusa.

\section{Il caso di Adua}

La narrazione, come anticipato, si sviluppa sull'intreccio di diverse conversazioni, scandite dalle tre sezioni in cui sono divisi i capitoli: "Adua," "Zoppe" e "Paternale." A queste si aggrovigliano altri racconti, come quelli del padre di Zoppe al figlio, e conversazioni, come quelle al telefono tra Adua e l'amica Lul. Il risultato è un mélange di mittenti e riceventi che comunicano oralmente, le cui voci sono romanzate come

${ }^{13}$ Astrid Erll e Ann Rigney hanno individuato tre ruoli che la letteratura ha nella produzione della memoria culturale: come "medium of remembrance," "object of remembrance" e come mezzo che ci permette di "osservare la produzione della memoria culturale" e di riflettere sui processi tramite cui si propaga, ma anche su questioni etiche ed epistemologiche (Erll/Rigney 112). Questi tre ruoli non rimangono distinti ma si mescolano tra di loro nel lavoro letterario. Per un approfondimento sulla definizione e lo sviluppo della memoria culturale: Erll, Memory.

${ }^{14}$ All'inizio del libro il vecchio Yacob le dice: "Tienila stretta quella curiosità e raccogli tutte le storie che puoi. Un giorno sarai la nostra voce che racconta [...] Sarai la voce della nostra storia che non vuole essere dimenticata" (Ghermandi 6). 
sintomatiche dei loro toni e lessici colloquiali. Un'ultima conversazione è quella aperta con i lettori, esortati al ricordo e alla trasmissione.

Scego inserisce spesso termini provenienti dal somalo, che nel testo sono segnalati dal corsivo: espressioni quali "Laabo dhegah, significa due pietre in italiano" (9); "conto su di te, abaayo, per conoscere ogni minimo dettaglio della mia casa che fu (10); "I corvi gracchiavano, i gor gor russavano" (45); "Zia Fardosa ha chiamato la migliore mammana per farvi il gudnisho" (92). Una precisazione è importante da fare qui; si potrebbe pensare che considerare queste espressioni come "orali" sia una scivolata dentro quel pregiudizio binomico africanità-oralità. In realtà, la stessa autrice, come cappello al glossario in fondo al libro, informa che l'alfabeto somalo è stato codificato solo nel 1974, e che durante la stesura di $A d u a$ ha preferito "restituire la sonorità di questa lingua presentando i termini somali come trascrizioni fonetiche pensate per i lettori italiani" (179). Le parole che si incontrano sono perciò storpiate per l'effetto descritto. Nella lista dei termini appare per esempio "Injeera," seguito dalla trascrizione corretta "Canjeero" e una nota: "pane tipico del Corno d'Africa" (180).

Il capitolo "Adua," che contiene la narrazione in prima persona della protagonista, si rifà ad un monologo, quello di Adua in presenza del "piccolo elefante di marmo che sostiene l'obelisco più piccolo del mondo" in Piazza della Minerva a Roma con cui Adua si sente accomunata dalla stessa sorte essendo anche l'animale un "errabondo" proveniente dall'Oceano Indiano (11). Adua fa infatti parte della prima generazione di migranti dalla Somalia, arrivati in Italia negli anni Settanta. Nel periodo in cui è ambientata la narrazione, Adua è ormai vecchia e racconta la sua infelice quotidianità con il marito, un ragazzo più giovane appartenente alla seconda ondata migratoria. Il loro rapporto viene descritto come "un amore di cartapesta" (169). Adua gli promette "una casa, una tetta, una minestra, un cuscino, un po' di quattrini, una speranza, una parvenza di qualsiasi respiro" in cambio di compagnia, di "un po' di attenzioni" (30). Il tacito patto tra i due consorti è quasi sempre rispettato. Adua sa che il marito chatta con altre ragazze che vivono in "posti dove i somali possono contare su un sussidio e un tetto a spese dello Stato" (89) e lo accetta perché sa che prima o poi se ne andrà da lei.

Adua consegna al compagno silenzioso la sua storia, creando al lettore l'illusione di essere, in fondo, quell'elefante e di raccogliere le parole della Vecchia Lira, parole che altrimenti "si sciolgono e si perdono" (11). Il fatto che l'unico interlocutore di Adua sia una statua ricorda le voci non ascoltate della migrazione e la sordità del paese ospitante che rimane indifferente alla molteplicità di situazioni simili a quella che viene narrata, pervasa da abusi, oltraggi, vessazioni, razzismo e rabbia. Nel libro, il portavoce di questo triste segmento di contemporaneità è il marito di Adua, chiamato da lei stessa "Titanic," dispregiativo con cui si indica uno dei tanti migranti che attraversano il Mediterraneo rischiando di affondare.

Il nostro dovere, come lettori, è quello di essere predisposti all'ascolto. Con postmemoria affiliativa Marianne Hirsch giustifica l'appropriazione di testimonianze da parte di chi non le ha ricevute direttamente da un genitore, e la conseguente creazione di un circuito di trasmissione più ampio di quello famigliare. L'affiliativa è quindi "il risultato della contemporaneità" e della connessione orizzontale tra membri di una stessa generazione attraverso "strutture di mediazione" in grado di "inglobare un collettivo più grande in una rete organica di trasmissione" (115). L'ammonimento al ricordo e alla divulgazione intarsia tutto il libro e sembra essere incarnato nella costante 
presenza dell'elefante, animale nelle cui grandi orecchie possono rifugiarsi i racconti dei protagonisti della diaspora e della migrazione transmediterranea contemporanea. Ad aprire il decimo capitolo, inoltre, lampeggia il titolo di un libro che Adua compra al supermercato: "Racconti le storie che hai come meglio puoi," che suona quasi come un comandamento da seguire (58).

Ma l'invito all'ascolto, dall'altra parte, rimanda anche all'insabbiamento del colonialismo, per anni rimasto un capitolo mancante dalla storia ufficiale della Repubblica; come ha sottolineato Del Boca, solamente negli anni settanta è stata riaperta la faglia della "notte coloniale" grazie allo storico Giorgio Rochat e al suo Il colonialismo italiano (1973), un "libro all'apparenza di poche pretese" che invece funge da apripista nell'ambito della storiografia contemporanea, essendo "lontano dalle interpretazioni romanzate od oleografiche" che puntellavano le pubblicazioni dei trent'anni successivi alla fine della guerra (Del Boca 590). Come Scego stessa esplicita nella "nota storica" in fondo al romanzo, i capitoli dedicati a Zoppe ricalcano alcuni eventi del colonialismo fascista che fanno da sfondo alla sua storia personale ma che vengono proposti in chiave critica: il riferimento al casus belli che Benito Mussolini utilizzò per invadere l'Etiopia, l'accoglienza delle truppe italiane a Port Said ad opera di Maria Uva e l'uso delle armi chimiche durante il conflitto etiope. Il fascismo è anche presente nell'architettura di Magalo, nel Faro, il munar, cui i fascisti avevano aggiunto una lama per trasformarlo in un fascio littorio e la frase "Per perpetua gloria di Roma" alla base (74), e nel cinema "con la sua austera architettura mussoliniana" (71).

Come si legge, la stessa Magalo, oltretutto, era rimasta fuori dai grandi avvenimenti; a Magalo "la storia [...] ci passava di sbieco" al contrario di Mogadiscio dove figure come Abdullahi Ciise (Abdullahi Issa Mohamud, 1922-1988) avevano attivato il processo d'indipendenza somala. Questo estraniamento sfocia da una parte nel ricordo nostalgico dell'AFIS nei racconti di alcuni, di solito "ex ascari o ex madame," che tramandano che "sotto gli italiani non si era vissuti poi così male" (74) e dall'altra nell'amnesia della memoria coloniale ad opera di coloro che l'avevano vissuta in prima persona ma che non avevano raccontato la verità, preferendo tacere, come Zoppe che, al massimo, "biascicava frasi, parole così vaghe che non spiegavano, non raccontavano" (74).

Il lettore viene a contatto con la storia di Zoppe, sconosciuta alla figlia e raccontata in prima persona, durante l'arco temporale compreso tra 1934 e il 1937 circa. Nel primo capitolo dedicato a lui si narra di un pestaggio ad opera di tre italiani a Roma, durante il quale, quasi privo di sensi, l'uomo ripensa alla sua decisione, contro il consiglio del padre Hagi Safar, di lavorare come traduttore, "ambasciatore della lingua" per gli italiani, "nuovi padroni" della Somalia, un impiego che gli avrebbe "fruttato un bel po' di quattrini”" (18):

Ogni giorno doveva tradurre, tradurre, tradurre e tradurre. Parole da decifrare ogni minuto, sospiri da segnalare ogni secondo, e poi tutte quelle maledette virgole da analizzare. Era un interprete, un mago quasi. [...] Parlava l'arabo, il somalo, il kiswahili, l'aramaico, il tigrino e una montagna di lingue piccole utili per la futura guerra. [...] L'italiano, invece, gliel'avevano insegnato i gesuiti. (18).

Trasferitosi a Roma, Zoppe, che "aveva preso quel trasferimento come un premio, un riconoscimento alla sua abnegazione, alla sua fedeltà," (19) invece che la città che aveva 
agognato, piena di "belle donne bionde" e "tanti amici con cui giocare a biliardo," ne aveva trovata una sporca e ostile allo straniero. Avversione palesata da gesti come gli sputi dei passanti che doveva evitare durante il tragitto da casa all'ufficio, cercando di compierlo nel minor tempo possibile. Si era anche reso conto di come il lavoro fosse particolarmente "doloroso" perché in quei documenti diplomatici che doveva tradurre c'era "odore di tradimento" (19). Dopo la prigionia a Regina Coeli causata da un dissidio mattutino di cui Zoppe non aveva colpa alcuna, viene comprato dal conte Celestino Anselmi, il quale lo porta ad Addis Abeba, dove si respira l'aria della guerra imminente, e successivamente a Mogadiscio, nella sua Somalia.

Zoppe si giustifica più di una volta con la figlia di non essere stato un collaborazionista, come se temesse il suo giudizio: "Dovresti ringraziarmi, ti ho dato il nome della prima vittoria africana contro l'imperialismo. Io, tuo padre, stavo dalla parte giusta. E non devi mai credere il contrario" (49). Nei ricordi di Adua, alla parola "collaborazionista" proferita "tremando" per riferirsi a un trafficante di mercenari, Zoppe veniva pervaso da "un tremolio che prendeva tutto il corpo, e lo faceva di gelatina" (72). E ancora: "Mio padre si agitava, sputava per terra, la bocca si riempiva di scongiuri e di improperi verso la figura che per lui incarnava il peccato più grande" (72). Quando Adua trova una foto del padre da giovane in compagnia "dei bianchi" Zoppe inveisce contro la figlia: "L'ho capito che metti in dubbio il mio essere stato un padre e un nazionalista. Pensi che stia recitando. Anche tua madre pensava la stessa cosa. Sempre a mettermi in dubbio [...] Credeva che fossi un traditore, un infame [...] C'è una bella differenza a tradire la patria e combattere la patria" (125). Emerge la famosa asserzione "traduttore traditore" che si riferisce a quel "tradimento" che l'interprete opera nel momento in cui "porta (i significati) attraverso" la barriera linguistica, perdendo e modificando quello che non può trasportare.

Ad Addis Abeba, un signore con "delle grosse orecchie da elefante," che disegna su un foglio l'immagine di un uomo "con il turbante blu e la faccia deformata da una cicatrice a forma di uovo" che scaraventa un giavellotto ad un elefante, appare in sogno a Zoppe (126-128). Solo successivamente si ricorderà di quella zia maga che gli aveva detto anni prima che "la nostra coscienza ha un viso" e che quell'uomo non era altro che il suo riflesso (129). Il sogno premonitore di Zoppe simboleggia il tradimento che tutta la vita egli preferirà nascondere. È solo verso la fine del libro che le visioni deliranti della guerra e il senso di colpa si fanno sempre più persistenti fino al momento dell'ammissione: "Mi sono venduto come un Giuda dei cristiani per trenta denari. Se quella gente morirà sarà anche per colpa mia" (151). A Massawa infatti, in uno scantinato umido, Zoppe è costretto a tradurre, senza tralasciare "nemmeno un respiro," l'accordo, tra due dignitari etiopi e il conte Anselmo, che avrebbe stipulato l'alleanza tra loro e l'Italia, contro gli etiopi stessi (162). In quell'occasione Zoppe si focalizza sul lavoro, nonostante sia preso dallo sconforto e dall'angoscia:

Doveva essere puntuale e preciso. Tradurre parola per parola. Non doveva giudicare. Aprire e chiudere la bocca era il suo compito. Nient'altro. Non doveva pensare. Non doveva intromettersi. Non doveva migliorare. Doveva solo aprire e chiudere la bocca [...] Zoppe in mezzo a loro si sentì solo. Attraversato dalle frecce avvelenate del tradimento. Ogni parola lo feriva. Ogni gesto lo oltraggiava (163-165).

La parola detta diventa importantissima, e la comunicazione orale costituisce un elemento testuale fondamentale in tutto il resoconto di Zoppe. Ne possono essere delle 
spie anche i riferimenti alla lingua come muscolo: la fisicità, in questo caso, si lega all'azione del parlare. Fisicità altrimenti negata dal soprannome Zoppe, che ricorda "zoppo", quindi storpio o malandato. ${ }^{15}$ Il conte Anselmo, subito dopo aver comprato Zoppe per le sue abilità di traduttore lo riceve nella sua abitazione. In quest'occasione non si fa riferimento all'azione del tradurre o al sapere linguistico, ma alla vigorosità della lingua:

"Mi ascolti, Zoppe, l'ho tirata fuori dai guai perché sono buono e perché lei mi serve. Mi mostri la lingua."

Zoppe ubbidì. Ormai non sapeva fare altro.

"Che bella lingua rossa, spessa. Mmm... mi piace. Mi sarà utile in Africa [...]" (87).

Ai capitoli "Adua" e "Zoppe" si interseca poi la "Paternale," sezione in cui il padre si rivolge alla figlia con toni aggressivi e autoritari. Ne sono esempi le seguenti citazioni: "è questo il modo di salutare i parenti, Adua? Con quella faccia? Sorridi. Che ce li hai a fare i denti se no? Sorridi” (32); "che cosa significa questo? Su, parla. Perché ti mando a scuola io, eh? Per leggere questa robaccia? Cos'è questo? Su, rispondi!" (81); "E anche se sei diventata grande sono capace di batterti fino a farti sanguinare l'anima" (107).

Già dal loro primo incontro, in cui Adua e la sorella Malika vengono prelevate dall'accampamento nomade in cui vivevano per essere trasferite a Magalo, il contatto affettivo con il padre viene meno quando la figlia lo cerca per ricevere conforto e spiegazioni e lui distoglie immediatamente lo sguardo: "capii in quel momento che mi stava rinnegando" (45). Il rapporto tra Adua e Zoppe è complesso, basato sull'odio recondito di Zoppe nei confronti di quella figlia non addomesticata che aveva procurato la morte durante il parto all'amata Asha la Temeraria, madre che Adua non conoscerà mai. Si trovano infatti diversi riferimenti alla rabbia di Zoppe nella Paternale: "Tua madre, quella troia, che è morta lasciandomi qui solo con il mio amore. Come si è permessa di morire? Eh? Come si è permessa? Maledetta femmina! E tu? Morirai pure tu? Hai gli stessi occhi suoi, non li sopporto!" (13); "l'unica cosa di veramente temerario che ha fatto tua madre è stato morire. Non ha fatto altro, solo morire" (49); "Dicono che è morta per amore, per amor mio. Hai mai sentito una sciocchezza così colossale? Morire per amore, come se fosse possibile. È morta perché era scema, tua madre, per fare un dispetto a me" (81). Adua, dall'altro canto, riflette spesso sulla relazione mancata con quel padre con cui non è mai andata d'accordo: "Avevamo caratteri forti, eravamo prime donne, mazzolati dalla vita" (27); "Non sapevo amarlo. E lui non sapeva amare me" (105).

Ma il legame tra Zoppe e Adua nasconde, a mio parere, un altro livello di lettura, ovvero la trasmissione verbale e non verbale del trauma del colonialismo da parte del padre nei confronti della figlia, racchiusa nel seguente passaggio:

Ma vedendo il tuo film ho pianto. Ho fallito in questa vita. Se ti ho permesso la mia stessa umiliazione significa solo che ho fallito. [...] Forse ti dovrei chiedere scusa. Ma non ci riesco. Certe parole non le so usare. Però una cosa te la posso dire, ho capito, guardando il film, quanto hai sofferto in questa vita. Alla fine io e te non siamo diversi, qualcuno ci

15 Alla domanda diretta di Emanuela, Zoppe risponde che il suo soprannome deriva da un infortunio: "Sì, perchè zoppico. Da piccolo ho avuto una brutta malattia, ma poi mi sono salvato (24). 
ha umiliato, schiacciato. Io sono rimasto sotto. Sono stato sconfitto. Forse tu sarai più fortunata. Forse (158-159).

L'ammissione di fallimento di Zoppe rimanda alla trasmissione inter-famigliare del dolore e della mortificazione nella forma della violenza contro la figlia, e anche alla comparazione di due situazioni analoghe ma appartenenti a due momenti della storia diversi. In primis quindi, la postmemory non è solo passaggio della conoscenza testimoniale ma anche l'imposizione di un modo di essere che Adua è costretta a subire, essendo il padre vittima e testimone allo stesso tempo, e di cui rimane lei stessa testimone e vittima. L'umiliazione si propaga inevitabilmente nel nucleo famigliare, attraverso "il linguaggio della famiglia, il linguaggio del corpo" nella "forma di sintomi” (Hirsch 112). L'unica speranza è nelle ultime parole riportate, in cui Zoppe introduce il "forse" di un futuro diverso. Solo dopo la morte del padre, Adua riuscirà a liberarsi di quella vergogna tramandata, simbolicamente materializzata nella stoffa blu del turbante appartenuto al padre che un gabbiano le strappa nel capitolo finale del libro, e che la libera dal "giogo" della redenzione (173).

La memoria, come l'ha definita Astrid Erll, è sempre in movimento (Erll, "Travelling” 15). Secondo Daniel Levy e Natan Sznaider, ${ }^{16}$ l'Olocausto ha permesso, tramite il superamento delle memorie tra eredi delle vittime ed eredi dei perpetratori, "il riconoscimento della storia dell'Altro," e quindi la creazione di una "memoria cosmopolita," resa possibile anche dal carattere globale della società contemporanea (465). Erll si spinge oltre introducendo le "memorie in viaggio" ("travelling memories"), definendo perciò la memoria come transculturale e slegata dalla nazione-stato. Questa accade perché la memoria si basa sul "traffico tra livelli individuali e collettivi del ricordo, circolando tra dimensioni sociali, mediali e semantiche" (Erll, "Travelling" 15). Il "viaggio" della memoria permette di creare connessioni e prospettive nuove, e implica la "curiosità - un'attenzione alle dimensioni del ricordare e del dimenticare che trascendono le frontiere" (ib.). In questo senso, la sorte comune condivisa da Zoppe con la famiglia di "ebrei bianchi," i Limentani, suggerisce il superamento della barriera etnica per una memoria condivisa (Scego 36).

I Limentani, infatti, sembrano essere gli unici a tollerare il colore della sua pelle, a guardarlo "con occhi umani" (16) nonostante gli ironici interventi della piccola Emanuela: "E da bambino eri così tanto marrone come sei adesso?" (24); "è la prima volta che viene a pranzo da noi un uomo marrone" (25). Durante il pestaggio iniziale, a Zoppe compaiono in sogno il padre e la figlia, mano nella mano. Nonostante non si sia ancora svelato il motivo, Zoppe si auspica che "il papà la porti lontano. Il più lontano possibile" (21). Solo poco dopo Emanuela racconterà con ingenuità le umiliazioni sopportate a scuola:

A scuola mi chiamano "assassina." Dicono che ho ammazzato Dio e che la mia famiglia va in giro a rubare i bambini. Ieri Graziella, quella grassa che non sa ancora l'alfabeto, mi ha tirato i capelli e mi ha chiamata "mangiaoche." Mi sono messa a piangere, perché ha tirato molto forte (26).

\footnotetext{
${ }^{16}$ Si veda: Levy/Sznaider. Per approfondimenti sulla memoria transnazionale: Rothberg; De Cesari/Rigney.
} 
A metà degli anni Trenta si respirava già il clima ostile che sarebbe poi sfociato nella promulgazione delle leggi razziali fasciste del 1938. Zoppe nella cella di Regina Coeli vede la proiezione di Rebecca, madre di Emanuela, e viene sopraffatto dalla certezza che "tutto stava cambiando", che "c'era un'ostilità che si faceva di giorno in giorno più palese, più sfacciata, più odiosa verso gli ebrei"(54). Compare anche l'interesse antropologico per le razze quando Zoppe si trova nella stanza con Bondi e Calamaro, i quali disquisiscono sui suoi caratteri somatici: "Non sono mica tutti uguali, sa? [...] Ci sono tipi diversi, per ogni regione. I capelli e il naso divergono enormemente. Dipende dal clima" (40); "Il signore è negro, ma ha tratti meno negroidi dei tipi antropologici che ho esaminato in Congo" (41). Anche il conte Anselmi sembra nutrire una certa curiosità nei confronti delle usanze "africane" che però hanno a che fare con la fisicità: "Sai danzare? [...] ah, che sciocco, praticherai le danze selvagge dei tuoi luoghi. [...] Quei balli dove siete nudi e agitati. Come bisce, per intenderci" (87).

In secondo luogo, suggerisce $A d u a$, quella del colonialismo è un'eredità che è insita nel tessuto sociale e culturale dell'Italia contemporanea e "viaggia" anche grazie alle forme di ri-mediazione culturale come il cinema. Il rapporto con il cinema guida i cambiamenti, le ambizioni e gli spostamenti di Adua. Inizialmente come spettatrice tra tante al vecchio cinema fascista restaurato dall'ex trafficante Idris Shangani, Adua sogna di scappare in Europa, in Italia, e di assaporare la "libertà" dei "baci sulla bocca, la mano nella mano, l'abbraccio appassionato" (74), insomma di vivere la dolcevita che il grande schermo ricreava per quelle donne somale destinate alle faccende di casa e all'allevamento della prole. Grazie al cinema Adua riesce ad accettare il suo trasferimento a Magalo e la finzione dei film sostituisce i sogni notturni sulla vita nomade che era stata forzata ad abbandonare: "Quella sera non sognai la mia boscaglia. Niente caprette, niente mamma, niente piogge, niente avvoltoi. C'era solo Maciste a cullarmi. I suoi capelli biondi come l'orizzonte. Dormii bene. E Magalo non mi sembrò più un posto orrendo" (80).

Un giorno, cresciuta ma ancora minorenne, Adua incontra "degli italiani" grazie ad Omar Genale, un esperto di "ogni tipo di traffico" illegale atto a sorpassare le restrizioni del regime (104). Con la promessa di diventare una stella di Cinecittà, Adua arriva a Roma. Si ritrova interprete di un film dal titolo Femina Somala dallo sfondo erotico, in cui corre nuda sulla spiaggia con una voce doppiata "tutta miele e languida" in contrasto con il suo "corpo solenne," per renderla più desiderabile e "far innamorare tutti gli uomini della terra" (90). La pellicola, fatta risalire al 1977 ad opera di Arturo Sposetti, è l'unico film che Adua girerà. Durante le riprese, è spesso costretta ad assumere pose esotizzanti, come quando è fatta sedere su un baobab di plastica "appollaiata come Jane di Tarzan" per la foto della locandina. La protagonista del film, impersonata da Adua, è Elo, una fotografa che "si spogliava nuda e si donava agli uomini come una cagna" (119). Uno di questi è Nick Tonno, il cooprotagonista, che è "un dannato finocchio" e per questo l'unico sul set a rispettarla (135). Ad Adua toccheranno diversi abusi a partire dalla straziante notte in cui la moglie del regista, Sissi, ordina al marito di possederla, e in cui avviene anche il brutale taglio con una forbice del filo dell'infibulazione, rito di iniziazione a quello che sarà il ruolo assunto da Adua nel film. Poco dopo, avviene l'incontro con il "marchese," un magnate che avrebbe distribuito il film, il quale le regala un bracciale d'oro in cambio della sua disponibilità a "vendersi" per una fellatio. 
La stereotipizzazione della donna nera ipersessualizzata e selvaggia nell'Italia degli anni Settanta in cui approda la giovane Adua è memore di quella perpetrata in epoca fascista. Nel racconto di Zoppe vengono menzionate le cartoline ritraenti donne nere in pose erotiche che circolavano tra i soldati per spingerli a partire per l'Africa. Orazio Civa, guardando una finestra di una casa di prostitute si riferisce a "quelle belle signorinotte $[. .$.$] quelle delle cartoline" (97). E poco oltre: "A Roma ho trovato certe fotine$ che proprio mi hanno invogliato" (99). ${ }^{17}$ L'umiliazione contro il corpo nero viene trasmessa all'interno del circuito letterario del romanzo tramite l'immagine (del cinema e delle cartoline), e quindi la trasmissione del trauma non solo tramite le parole ma anche attraverso la materialità degli oggetti e altri media.

In conclusione, come ho cercato di dimostrare, mi sembra produttivo riferirsi all'oralità per due motivi. Da una parte per eliminare gradualmente la tradizionale concezione del testo postcoloniale italiano come un testo che riprende, riafferma e rivendica una "lingua africana" in opposizione a quella metropolitana dell'italiano. Un procedimento che non credo possa essere attribuito ai nostri autori di seconda generazione, i quali, semmai, si appropriano di convenzioni di genere, come l'inserimento di un formulario "indigeno," scaturite dal contesto anglofono e francofono nell'ambito del romanzo postcoloniale. Dall'altra, perché orale è tutta la struttura narrativa di libri che come Adua ripercorrono la trasmissione della memoria tra generazioni (di padre in figlio), intessendo vincoli di affiliazione e parallelismi tra momenti storici (epoca fascista e contemporaneità) e contesti geografici (Etiopia, Somalia, Italia) diversi, intrecciando l'ascolto con la parola, il detto con il non detto, i testimoni con coloro che accoglieranno la memoria, la memoria con la storia ufficiale, la storia con il dibattito pubblico, attraverso il cinema, la letteratura e altri media.

Adua racconta che il giovane marito ogni tanto le cantava delle poesie d'amore che "la sua povera madre paralitica gli aveva insegnato una notte, per distrarlo dalle bombe che cadevano come grandine sul loro capo scoperto" (116). La trasmissione unisce anche Zoppe al padre Hagi Safar, il quale racconta le storie degli antenati, "che odoravano di cannella e cardamomo" e "che legavano quel vecchio padre all'amatissimo figlio" (161). Filtrare la letteratura postcoloniale italiana attraverso il setaccio dei memory studies permette di concentrarsi sul sovrapporsi non casuale della lingua somala al racconto di storie della tradizione e ai ricordi dell'infanzia. Sostituire il nesso africanitàoralità con quello di trasmissione-oralità significa ridimensionare quella componente "altra" (cosa c'è di africano, e quindi di "puro" e "autentico" nel testo) che tende a valorizzare la portata documentaristica della letteratura italiana postcoloniale a discapito della sua letterarietà; significa, quindi, riposizionarla nel canone della letteratura italiana tout-court.

\footnotetext{
${ }^{17}$ Per un approfondimento: Ponzanesi.
} 


\section{Bibliografia}

Ahmed, Siraj. "Notes from babel: Toward a colonial history of comparative literature". Critical Inquiry 39.2 (2013): 296-326.

Ali Farah, Cristina Ubax. Madre Piccola. Milano: Frassinelli, 2007.

Assmann, Aleida. "History, Memory, and the Genre of Testimony". Poetics Today 27 (2006): 261-273.

Assmann, Jan. "From Moses the Egyptian: The Memory of Egypt in Western Monotheism". Ed. Jeffrey Olick, Vered Vinitzky-Seroussi/Daniel Levy. The collective memory reader. Oxford: Oxford UP, 2011. 209-215.

Bandia, Paul. Translation as Reparation. Writing and translation in postcolonial Africa. Oxon: Routledge, 2008.

Bandia, Paul. "Postcolonial literary heteroglossia: a challenge for homogenizing translation”. Perspectives 20.4 (2012): 419-431.

Barber, Karin. The anthropology of texts, persons and publics. Cambridge: Cambridge UP, 2007.

Bhabha, Homi. The location of culture. New York; Routledge, 1994.

Chamoiseau, Patrick. "Que faire de la parole? Dans la tracée mystérieuse de l'oral à l'écrit". Écrire la 'parole de nuit.' La nouvelle littérature antillaise. Ed. Ralph Ludwig. Paris: Gallimard, 1994. 151-158.

Comberiati, Daniele. La quarta sponda. Scrittrici in viaggio dall' Africa coloniale all'Italia di oggi. Roma: Edizioni Pigreco, 2007.

Comberiati, Daniele. Scrivere nella lingua dell'altro: la letteratura degli immigrati in Italia (1989-2007). Oxford: Peter Lang, 2010.

Crownshaw, Rick. "Trauma Studies". The Routledge Companion to Critical and Cultural Theory. Ed. Paul Wake/Simon Malpas. London: Routledge, 2013. 167-176.

De Caldas Brito, Christiana. Amanda Olinda Azzurra e le altre. Roma: Lilith Edizioni, 1998.

De Cesari, Chiara e Ann Rigney (Ed.). Transnational Memory: Circulation, Articulation, Scales. Berlin: De Gruyter, 2014.

Del Boca, Angelo. "Il colonialismo italiano tra miti, rimozioni, negazioni e inadempienze”. Italia contemporanea 212 (1998): 589-603.

Erll, Astrid/Ann Rigney. "Literature and the production of cultural memory: introduction". European Journal of English Studies 10.2 (2006): 111-115.

Erll, Astrid. "Travelling Memory". Parallax 17.4 (2011): 4-18.

Erll, Astrid. Memory in Culture. Basingstoke: Palgrave Macmillan, 2011.

Foucault, Michel. Language, counter-memory, practice: Selected essays and interviews. Ithaca: Cornell University Press, 1980.

Ghermandi, Gabriella. Regina di fiori e di perle. Roma: Donzelli Editore, 2011.

Ghosh, Amitav. "The Diaspora in Indian Culture". Public Culture 2.1 (1989): 73-78.

Gikandi, Simon. “Editor’s Column - Provincializing English”. PMLA 129.1 (2014): 7-17.

Hirsch, Marianne. “The Generation of Postmemory”. Poetics Today 29.1 (2008):103128.

Julien, Eileen. "Il romanzo africano: un genere "estroverso". Il Romanzo IV. Ed. Franco Moretti. Milano: Einaudi, 2003. 155-179. 
Levy, Daniel/Natan Sznaider. "From 'Memory Unbound: The Holocaust and the Formation of Cosmopolitan Memory"'. The collective memory reader. Ed. Olick, Jeffrey K./Vered Vinitzky-Seroussi/Daniel Levy. Oxford: Oxford University Press, 2011. 465-467.

Lionnet, Françoise. “'Logiques métisses:' Cultural Appropriation and Postcolonial Representations". Teaching Postcolonial and Commonwealth Literatures 19.3 (1992): 100 120.

Lombardi-Diop, Cristina/Caterina Romeo (Ed.). Postcolonial Italy: challenging national homogeneity. New York: Palgrave Macmillan, 2012.

Lombardi-Diop, Cristina/Caterina Romeo. "The Italian Postcolonial: A Manifesto". Italian Studies 69.3 (2014): 425-33.

Nora, Pierre. "Between Memory and History: Les Lieux de Mémoire". Representations 26 ([1984]1989): 7-25.

Olick, Jeffrey, Vered Vinitzky-Seroussi/Daniel Levy (Ed.). The collective memory reader. Oxford: Oxford UP, 2011.

Ong, Walter J. Orality and Literacy: The Technologizing of the Word. New York: Routledge, 1982.

Sharmani, Patricia Gabriel. "The heteroglossia of home". Journal of Postcolonial Writing 41.1 (2005): 40-53.

Ponzanesi, Sandra. "Beyond the Black Venus: Colonial Sexual Politics and Contemporary Visual Practices". Italian Colonialism: Legacy and Memory. Ed. Derek Duncan and Jacqueline Andall. Bern: Peter Lang, 2005.

Quaquarelli, Lucia. "Decentrare la lingua. Alcune considerazioni sul caso italiano". Comparative Studies in Modernism 11 (2017): 73-84.

Romeo, Caterina. "Italian Postcolonial Literature". California Italian Studies 7.2 (2017): $1-44$.

Romeo, Caterina. "Vent'anni di letteratura della migrazione e di letteratura postcoloniale in Italia: un excursus". Bollettino di italianistica 8.2 (2011): 381-408.

Rothberg, Michael. "From Gaza to Warsaw: Mapping Multidirectional Memory". Criticism 53 (2011): 523-548.

Scego, Igiaba. Adua. Firenze: Giunti, 2015.

Smith, Andrew. "Migrancy, Hybridity and Postcolonial literary studies". The Cambridge Companion to Postcolonial Literary Studies. Ed. Neil Lazarus. Cambridge: CUP, 2004. 241-261.

Wieviorka, Annette. “The Witness in History”. Poetics Today 27 (2006): 385-397. 